\title{
REVIEW
}

\section{Complex Regional Pain Syndrome: A Comprehensive Review}

\author{
Samantha-Su Taylor · Nazir Noor (D) - Ivan Urits · Antonella Paladini • \\ Monica Sri Sadhu • Clay Gibb · Tyler Carlson · Dariusz Myrcik • \\ Giustino Varrassi · Omar Viswanath
}

Received: January 11, 2021 / Accepted: June 3, 2021 / Published online: June 24, 2021

(C) The Author(s) 2021, corrected publication 2021

\section{ABSTRACT}

Complex regional pain syndrome (CRPS) is a chronic pain condition often involving hyperalgesia and allodynia of the extremities. CRPS is divided into CRPS-I and CRPS-II. Type I occurs when there is no confirmed nerve injury. Type II is when there is known associated nerve injury. Female gender is a risk factor for developing CRPS. Other risk factors include

S.-S. Taylor · M. S. Sadhu · T. Carlson .

O. Viswanath

University of Arizona College of Medicine-Phoenix, Phoenix, AZ, USA

\section{N. Noor $(\bowtie)$}

Department of Anesthesiology, Mount Sinai

Medical Center, 4300 Alton Road, Miami Beach,

FL 33130, USA

e-mail: naz9noor@gmail.com

I. Urits

Southcoast Physician Group Pain Medicine,

Southcoast Health, North Dartmouth, MA, USA

I. Urits · O. Viswanath

Department of Anesthesiology, Louisiana State

University Health Shreveport, Shreveport, LA, USA

A. Paladini

Department of MESVA, University of L'Aquila,

67100 L'Aquila, Italy fibromyalgia and rheumatoid arthritis. Unfortunately, the pathogenesis of CRPS is not yet clarified. Some studies have demonstrated different potential pathways. Neuropathic inflammation, specifically activation of peripheral nociceptors of C-fibers, has been shown to play a critical role in developing CRPS. The autonomic nervous system (ANS) is involved. Depending on whether it is acute or chronic CRPS, norepinephrine levels are either

\section{Gibb}

Midwestern University Chicago College of

Osteopathic Medicine, Chicago, IL, USA

\section{Myrcik}

Department of Internal Medicine, Medical

University of Silesia, 42-600 Katowice, Bytom, Poland

G. Varrassi

Paolo Procacci Foundation, Via Tacito 7, Rome, Italy

O. Viswanath

Valley Pain Consultants-Envision Physician Services, Phoenix, AZ, USA

O. Viswanath

Department of Anesthesiology, Creighton

University School of Medicine, Omaha, NE, USA 
decreased or increased, respectively. Some studies have suggested the importance of genetics in developing CRPS. More consideration is being given to the role of psychological factors. Some association between a history of depression and/or post-traumatic stress disorder (PTSD) and the diagnosis of CRPS has been demonstrated. Treatment modalities available range from physical therapy, pharmacotherapy, and interventional techniques. Physical and occupational therapies include mirror therapy and graded motor imagery. Medical management with non-steroidal anti-inflammatory drugs (NSAIDs) has not shown significant improvement. There have been supporting findings in the use of short-course steroids, bisphosphonates, gabapentin, and ketamine. Antioxidant treatment has also shown some promise. Other pharmacotherapies include lowdose naltrexone and Botulinum toxin A (BTXA). Sympathetic blocks are routinely used, even if their short- and long-term effects are not clear. Finally, spinal cord stimulation (SCS) has been used for decades. In conclusion, CRPS is a multifactorial condition that still requires further studying to better understand its pathogenesis, epidemiology, genetic involvement, psychological implications, and treatment options. Future studies are warranted to better understand this syndrome. This will provide an opportunity for better prevention, diagnosis, and treatment of CRPS.

Keywords: Algoneurodystrophy; Causalgia; Complex regional pain syndrome (CRPS); Posttraumatic stress disorder (PTSD); Reflex sympathetic dystrophy (RSD); Sudeck atrophy

\section{Key Summary Points}

Complex regional pain syndrome (CRPS) is a chronic pain condition often involving hyperalgesia and allodynia of the extremities.

CRPS is divided into CRPS-I and CRPS-II.

Risk factors are female gender, fibromyalgia, rheumatoid arthritis, neuropathic inflammation, autonomic nervous system alterations, and psychological factors.

Main treatments are physical therapy, pharmacotherapy, and interventional techniques.

Conclusions: CRPS is a multifactorial condition with uncertain treatment options.

\section{DIGITAL FEATURES}

This article is published with digital features, including a summary slide, to facilitate understanding of the article. To view digital features for this article go to https://doi.org/10.6084/ m9.figshare.14717196.

\section{INTRODUCTION}

Complex regional pain syndrome (CRPS) is a chronic pain condition characterized by hyperalgesia and allodynia, commonly involving the limbs. It frequently develops after extremity trauma or surgery [1-3]. The pathophysiology underlying the development of CRPS is still being explored, but the disorder is believed to result from central and peripheral nervous system dysfunction [4]. The definition and diagnosis of CRPS have evolved over the years. Historically, this syndrome was known as "reflex sympathetic dystrophy", "causalgia", "algoneurodystrophy", or "Sudeck atrophy" [5-7]. CRPS is categorized into two types: CRPS-I occurs in patients without confirmed nerve 
injury, while CRPS-II occurs in patients with associated nerve damage [6]. However, providers often rely on clinical findings for diagnosis, as there are no specific diagnostic tests available [8]. CRPS includes a variable clinical presentation and disease progression. Many patients experience a severely impacted quality of life and poor prognosis despite treatment $[6,9]$. Early diagnosis and initiation of treatment is critical to limit disease progression and improve patients' quality of life. The purpose of this review is to provide an update on the current literature regarding epidemiology, pathophysiology, and current therapy for CRPS.

\section{COMPLIANCE WITH ETHICS GUIDELINES}

It is important to note that this article is based on previously conducted studies and does not contain any studies with human participants or animal performed by the authors without a previous Ethics Committee approval.

\section{EPIDEMIOLOGY}

To date, the largest population-based study of CRPS in the United States was published in 2016. The retrospective analysis of the Nationwide Inpatient Sample database from 2007 to 2011 found that out of 33,406,123 total patients in the study, 22,533 patients or $0.07 \%$ were discharged with a diagnosis of CRPS [10]. Population factors that were associated with CRPS included female gender, Caucasian race, higher median household income, and presence of comorbidities such as depression, drug abuse, and headache. Conditions such as obesity, diabetes, hypothyroidism, and anemia were associated with lower rates of CRPS [10]. A smaller study that analyzed $6,575,999$ patients in the Truven MarketScan Commercial and Medicare Supplemental database from 2000 to 2012, found the overall prevalence of CRPS to be slightly higher, $1.2 \%$, and positively correlated with female gender, long-term disability, and multiple pain diagnoses [11]. Additionally, a separate analysis of the same database demonstrated a significant increase in healthcare utilization and cost among CRPS patients 1 year prior to and at the time of diagnosis [12].

It is important to note that CRPS is not a diagnosis exclusive to adults but there is certainly a paucity of evidence in regards to diagnosis and treatment of the pediatric population [13]. A 2021 systematic review by Karri et al. focuses CRPS in the pediatric population and it considers neuromodulation, a known treatment modality in adults, for pediatric patients [13]. The primary goals of treatment of CRPS in the pediatric population are pain relief and improving all domains of functioning in order to improve the patient's quality of life [14]. Therapy includes intensive physical therapy combined with cognitive behavioral therapy intervention [14]. Among other treatments in the standard of care approach, pediatric patients may be considered for neuromodulation interventions, such as spinal cord stimulation (SCS) or dorsal root ganglion stimulation (DRG) devices [13]. Karri et al. recommend careful consideration of pubertal growth spurt prior to device lead placement given the possibility of inferior lead migration with physiologic growth in patients with SCS devices or foraminal extrusion in patients with DRG devices [13].

\section{Risk Factors}

Extremity injuries, such as fractures or sprains, surgery, and carpal tunnel syndrome have been the most commonly reported inciting events leading to CRPS $[1-3,15]$. In a recent analysis of 1043 patients with CRPS, the most common primary causes were fractures (42\%), blunt traumatic injuries excluding fractures (e.g., sprains) $(21 \%)$, surgery $(12 \%)$, and carpal tunnel syndrome $(7 \%)$, while $7 \%$ had no clear precipitating event [1]. However, the overall prevalence of CRPS associated with these inciting events remains relatively low. Crijns et al. found that out of 59,765 patients treated for distal radius fractures, $0.19 \%$ were diagnosed with CRPS [16]. A similar more recent study noted a $0.64 \%$ incidence of CRPS after 172,194 patients receiving surgical treatment for distal radius fractures [3]. CRPS had previously been reported 
as a common complication of Dupuytren contracture, but a very recent analysis of a nationwide database found the overall incidence to be $0.31 \%$ [17].

Multiple studies have demonstrated CRPS to be much more prevalent in women, with estimates of 2-4 times the rate in men [1, 3, 10-12, 18]. Van Velzen et al. further investigated these sex-related differences, finding that male CRPS patients were more likely to suffer from depression and kinesiophobia, and use passive pain coping strategies [18]. Fibromyalgia is strongly and independently associated with a diagnosis of CRPS, increasing the risk up to 2.5 times that of controls $[16,19]$. Other musculoskeletal conditions, such as rheumatoid arthritis, may also increase the risk [20]. Although CRPS and other autonomic disorders have been anecdotally reported as adverse events after human papillomavirus (HPV) vaccination, review of published reports and a small population-based case series found no statistically significant correlation [21-23].

\section{CRPS PATHOPHYSIOLOGY}

Despite being studied for over two decades, the exact pathogenesis of CRPS is still incompletely understood $[9,24]$. CRPS is characterized by an abnormal tissue response to injury as well as increased sensitization of the peripheral and central nervous systems with accompanying inflammatory changes and autonomic dysregulation [7]. Genetic and psychological contributions are also believed to play a role in the progression of CRPS [7].

The clinical course of CRPS is believed to develop in two phases: the acute, or warm phase, which involves the release of pro-inflammatory modulators, and the chronic, or cold phase, which is characterized by the activation of keratinocytes, fibroblasts, and osteocytes $[9,24]$.

\section{Inflammation}

CRPS is characterized by both a pro-inflammatory immune response and impaired neuropeptide signaling [25]. Inflammation is an expected outcome after stroke, surgery, or tissue trauma; however, the activation of the innate immune system is amplified and persistent in CRPS patients [9]. This innate immune system activation triggers the proliferation of keratinocytes and the release of proinflammatory cytokines including interleukin-6 (IL-6), IL-1 $\beta$ and tumor necrosis factor- $\alpha$ (TNF- $\alpha$ ) [26]. These cytokines trigger an immune cascade that results in histamine-induced vasodilation, causing the redness, swelling, pain, and warmth that is characteristic of the acute phase of CRPS $[26,27]$. Pro-inflammatory cytokines also activate osteoblasts and osteoclasts, resulting in the rapid bone turnover and osteoporotic changes that are characteristic of the chronic phase of CRPS [4, 24, 28].

Expanded populations of CD4+ and CD8+ lymphocytes in CRPS patients were documented in multiple studies, suggesting an antigen-mediated T lymphocyte response [29, 30]. A recent study examining serum levels of pro- and anti-inflammatory cytokines in the serum of CRPS patients also identified IL-37 and GM-CSF as novel biomarkers in the immune response [29]. Decreased serum levels of IL-37 were found, indicating a suppression of the immune response through the activation of IL-10 and regulatory $\mathrm{T}$ lymphocytes [27]. In contrast, an increase of GM-CSF was noted, highlighting a balance between pro- and anti-inflammatory cytokines in the pathogenesis of CRPS, with a predominantly pro-inflammatory state $[27,29]$.

Neuropathic inflammation is also believed to play a central part in the development of CRPS. The activation of peripheral nociceptors of C-fibers results in the transmission of pain signals afferently towards the dorsal ganglia and efferently towards the affected tissue [31]. Proinflammatory neuropeptides including Substance $\mathrm{P}$ and calcitonin gene-related peptide (CGRP) are produced from this backward transmission [31]. A study of CRPS patient skin biopsies confirmed that Substance P and CGRP were bound to their receptors on keratinocytes, resulting in keratinocyte proliferation and neurogenic inflammatory changes with subsequent hyperalgesia and allodynia [32]. A recent case study noted that $A-\alpha$ nerve fibers of the peripheral nerves of a CRPS patient were 
significantly degenerated, but noted that A- $\delta$ nerve fibers were spared [33]. The study hypothesized that increased A- $\delta$ nociceptive activity may explain the allodynia and hyperalgesia seen in CRPS patients [33].

\section{Autonomic Nervous System}

An imbalance in the autonomic nervous system of CRPS patients often leads to clinical manifestations such as skin color changes, increased heart rate, decreased heart rate variability, low cardiac output, and excessive sweating [34]. Subsequent studies noted that the autonomic imbalance of CRPS is explained by the increased expression of $\alpha-1$ adrenergic receptors on keratinocytes and nociceptors [34]. Under normal circumstances, sympathetic activation results in the release of catecholamines such as norepinephrine, which bind $\alpha-1$ adrenergic receptors causing vasoconstriction [35]. CRPS patients, however, were found to have decreased norepinephrine levels in their affected limb but increased overall systemic catecholamine expression [34].

In the acute phase of CRPS, sympathetic nervous system activity is decreased, leading to lower circulating levels of norepinephrine [34]. As a result, peripheral $\alpha-1$ adrenergic receptors are upregulated and sensitized [5]. This results in vasodilation and increased blood flow to the CRPS affected limb, leading to warmth and erythema $[5,35]$. Similarly, during the chronic cold phase of CRPS, the prolonged release of proinflammatory cytokines, including endothelin-1, results in excessive sympathetic nervous system outflow leading to increased norepinephrine levels and decreased $\alpha-1$ adrenergic receptor expression, culminating in vasoconstriction and the development of a cold, blue, clammy limb $[5,35]$.

Consistent with the above findings, a study observed that the upregulated expression of $\alpha-1$ adrenergic receptors was found to be a source of pain in CRPS patients after phenylephrine, an $\alpha$ 1 analog, was injected intradermally [36]. The patients who reported hyperalgesia after the phenylephrine injections expressed a greater number of cutaneous $\alpha$-1adrenergic receptors
[36]. Furthermore, CRPS II patients demonstrated increased $\alpha-1$ adrenergic receptor expression, as did patients with acute CRPS as compared to those with chronic CRPS [36].

Other recent studies have shown that the sympathetic nervous system is involved in local pain pathways via the sprouting of sympathetic fibers into the DRG after spinal nerve ligation [37]. In a rat model, the knockdown of sympathetic nervous system pathways resulted in drastically reduced mechanical pain behaviors as well as decreased sprouting of sympathetic nerve fibers into the DRG, providing clear evidence of a sympathetic-sensory link in CRPS [38].

\section{Autoimmunity}

The evidence for autoimmunity in CRPS comes from both prior studies which have observed elevated levels of autoantibodies in the serum, skin, and tissues of CRPS patients, as well as from animal models [39]. It is believed that autoantibodies produce pain in CRPS by sensitizing nociceptors [40].

Recent mice models studied the effects of transferring serum IgG from CRPS patients into mice with hind paw incisions [40]. It was found that mice who received serum IgG from CRPS patients displayed increased hypersensitivity to painful mechanical stimuli like cold and heat, but not to painful tactile stimulation [40]. Additionally, it was found that CRPS patients who endorsed greater pain had higher IgG antibody titers than patients who endorsed lower pain levels [40]. These studies illustrated that the IgG antibodies found in the serum of CRPS patients sensitize $\mathrm{A}$ and $\mathrm{C}$ nociceptors, maintaining the painful hypersensitivity that is characteristic of persistent CRPS [40].

Experimental fracture models studying CRPS also found increased levels of IgM antibodies in the skin and spinal tissue of rats, which were believed to cause increased nociceptive sensitization [25]. CRPS related IgM antibodies are postulated to lead to pain via three main mechanisms: direct interaction with their targets, complement activation, and the deposition of additional antibodies [41]. 
Landmark experiments using in vitro beating cardiomyocytes found that patients with longstanding CRPS had elevated serum levels of functionally active autoantibodies with $\alpha-1$ adrenergic, $\beta-2$ adrenergic, and M2 muscarinic agonist activity $[42,43]$. It was also found that while such antibodies were commonly present in the serum of CRPS patients, they were generally not found in patients with other chronic pain conditions [42]. Emerging evidence delineating the role of autoimmunity in CRPS provides hope for new therapeutics interventions targeting autoimmunity $[39,41]$.

\section{GENETIC/EPIGENETIC FACTORS}

\section{Genetic Factors}

The relationship between genetic factors and CRPS has been studied for many years with no concrete genetic link found. There is a suspected link due to familial aggregation and similar findings among CRPS patients. The human leukocyte antigen system (HLA) was the primary focus of studies because the genes in that system were found to be the most significantly upregulated (HLA-DRB1) and downregulated (HLA-DQB1) [44]. The abnormal regulation of these genes suggests that the immunes system, and more specifically the adaptive immune response, is a driving factor behind the development of CRPS. Among the HLA family, the expression of HLA-DQB1 was increased among CRPS patients [44, 45]. HLADQB1 is expressed by a subgroup of immune cells which includes B cells, activated T cells and macrophages. One study theorizes that this genetic association between HLA-DQB1 and CRPS can be compared to that of the association between HLA-DQ8 and celiac disease. The HLA gene fulfills the role of binding gluten-peptides on antigen-presenting cells. Gluten-specific CD4+ T-cells in the lamina propria respond to these peptides and enhance cytotoxicity of the locally present lymphocytes. Gluten peptides gain an enhanced affinity to bind to gliadin T-cell epitopes, which promotes a downstream inflammatory effect. The proposed mechanism of celiac disease is that once the threshold of gluten on T-cells has been reached, a self-amplifying loop that causes a continuous inflammatory response is established. The exact nature of the inflammatory response in CRPS is unknown, but this offers an interesting avenue that can be pursued [46]. A recent study explored the correlation behind CRPS and exosomes. The study found changes in gene expression among human cells following the uptake of exosomes enriched in miR-939 [47]. There are many gaps in our knowledge about exosomes and their relationship to pain and more specifically CRPS. As more studies on the subject are published, we hope to fill in some of those gaps.

\section{Epigenetic Factors}

The study of how epigenetics influences pain and more specifically CRPS is very young with limited sources. A study of post-war amputee victims was conducted in which DNA methylation was the epigenetic factor that was observed. CpG sites which contain the DNA sequence cytosine-phosphate-guanine nucleotides were the sites of interest, these sites are known to be affected by genetic and environmental factors such as trauma, smoking, and diet. The sites also suppress transcriptional activity [45]. The study revealed that $48 \mathrm{CpG}$ sites were statistically significant in how they were methylated. All but seven of the 48 sites were hypomethylated compared to the nonCRPS patients and a substantial amount of the 48 sites were related to immune function. Over one-third of the CRPS patients exhibited a higher-than-normal level of antineuronal antibodies [45]. The apparent commonalities among both genetic and epigenetic factors are found within the characteristics of the immune system. Based on the current findings, it would suggest that a focus on the immune system and inflammatory system and their relationship to CRPS would be the best approach for further understanding. 


\section{PSYCHOLOGICAL FACTORS}

The results of studies concerning the role of psychological factors in the development of CRPS are inconclusive. More dated studies have suggested the association does not exist; however, more recent studies have suggested that there is a connection between psychological factors and pain outcomes in patients with CRPS [48]. Few studies have been conducted on this topic and for us to truly under the relationship more research is needed.

\section{Depression}

It has been suggested that psychological disorders, such as depression, may contribute to the development of CRPS [6]. A study conducted comparing CRPS patients, major depressive disorder (MDD) patients, and a control group suggested that psychological profiles do not predispose the individual to the development of CRPS. The psychological profile may be secondary to the pain or contribute to the chronicity of the pain however [48]. The anxiety and depression that MDD patients were experiencing involved emotional dysregulation but this differed from the mechanism of depression in CRPS patients. This difference between CRPS and MDD patients may suggest that CRPS patients have an intact emotional regulation and that their depression is not the same as the mental disorder depression [48]. Though the causal relationship remains unclear, it is known that depression is one of the most common psychiatric diagnoses among CRPS patients and thus each patient should be observed for depressive symptoms.

\section{PTSD}

It has been suggested that there may be a link between post-traumatic stress disorder (PTSD) and CRPS. A study was conducted where 152 patients with CRPS, 55 control with chronic pain and 55 age- and sex-matched healthy individuals were evaluated for PTSD; 38\% of the CRPS patients (58), $10 \%$ of the non-CRPS pain patients (six), and $4 \%$ of the healthy individuals (four) met the criteria for PTSD. Of the 58 CRPS patients who met the PTSD criteria, 86\% (50) had PTSD symptoms prior to the CRPS diagnosis; $14 \%$ of the patients (eight) developed PTSD during the course of CRPS [49]. From the limited scope of this study, we can infer that PTSD is more prevalent in CRPS patients. It should be noted this was one of the few studies of its kind that we could find. More research into this subject would allow us to yield a more concrete conclusion.

\section{Neuropsychological}

CRPS seems to cause complex neurological changes that can affect a patient's way of life similar to that of a brain lesion. These changes include ownership of the affected body part, distortion of size, negative feelings toward the affected body part, and deficits in both lateralized spatial and non-spatial-lateralized cognitive functions [50].

\section{DIAGNOSIS CRITERIA}

In 1993, a meeting in Orlando, Florida, held by the International Association for the Study of Pain (IASP), developed a consensus terminology and standardized diagnostic criteria to improve clinical recognition of CRPS [51]. Since the IASP criteria for CRPS, studies have suggested its lack of specificity, resulting in many false positives that contribute to overdiagnosis and unnecessary and inappropriate treatments [51]. One reason for this shortcoming in specificity by the IASP criteria was its failure to incorporate motor and trophic features commonly associated with CRPS [51]. Ten years later in 2003, another international consensus meeting was held in Budapest, Hungary, to review the issues with the diagnosis of CRPS with the goal of recommending improvements to the IASP criteria [51]. This came to be known as the Budapest Criteria, which is currently the accepted diagnostic criteria for the diagnosis of CRPS.

The Budapest Criteria requires (1) continuing pain disproportionate to any inciting event, (2) reports by the patient of at least one symptom in three of the four categories of sensory, 
vasomotor, sudomotor/edema, and/or motor/ trophic, (3) must display at least one sign at the time of evaluation in two or more of the four categories listed previously, and (4) there is no other diagnosis that better explains the patient's signs and symptoms [51]. Sensory symptoms include reports and/or presence of hyperesthesia and/or allodynia. Vasomotor symptoms include reports and/or presence of temperature asymmetry and/or skin color changes and/or skin color asymmetry. Sudomotor/edema symptoms include reports and/or presence of edema and/or sweating changes and/or sweating asymmetry. Motor/trophic symptoms include reports and/or presence of decreased range of motion and/or motor dysfunction (weakness, tremor, dystonia) and/or trophic changes (hair, nail, skin) [51].

Furthermore, diagnostic subgroups demonstrated that $47.7 \%$ as opposed to $74.4 \%$ had lower extremity involvement with CRPS when compared to non-CRPS, respectively. Among this subgroup, CRPS also involved the right side in 50.5\%, while non-CRPS involved 57.1\% [51].

\section{CURRENT TREATMENT}

As symptoms of CRPS exhibit a variable progression over time, early initiation of therapy is integral to patient prognosis, with the goals of restoring limb functionality, decreasing pain, and improving quality of life $[52,53]$. This often requires a multidisciplinary approach involving patient education, physical and occupational therapy, psychiatry, and pain medicine specialists, along with pharmacological and surgical interventions.

\section{Physical and Occupational Therapy}

Physical and occupational therapies are key initial components of treatment to help CRPS patients overcome their fear of pain and kinesophobia. Several therapeutic modalities have been studied such as massage, electrotherapy, acupuncture, contrast baths, biofeedback, isometric strengthening exercise, counter strain, and gentle range of motion. Among various physiotherapy interventions, a Cochrane review of 18 RCTs identified graded motor imagery and mirror therapy as providing the greatest rehabilitation benefit, significantly improving pain and quality of life, although the quality of evidence was very low $[54,55]$. A recent randomized comparative effectiveness trial evaluated the use of a modified graded motor imagery program in women at risk for developing CRPS after distal radius fracture treated with cast immobilization [56]. However, larger and higher-quality RCTs are still needed.

\section{Pharmacotherapy}

A variety of medications have been utilized for symptomatic pain management of CRPS, with the primary goal to enable patients to participate in rehabilitation regimens. Traditionally, non-steroidal anti-inflammatory drugs (NSAID) and corticosteroids have been used to target the pain and inflammation underlying CRPS in both adults and pediatric patients [14, 57]. However, current studies have demonstrated no evidence to support the use of NSAIDs [58]. A recent 2020 double-blind RCT of 91 patients with distal radius fractures found a slightly lower incidence of CRPS in patients who took a 7-day course of $500 \mathrm{mg}$ aspirin, but it was not statistically significant [59]. One high-quality RCT of 60 patients demonstrated a positive effect of oral prednisolone compared to the NSAID piroxicam on CRPS symptoms following stroke, although the study groups exhibited some baseline differences [60]. However, highdose corticosteroids may not be beneficial for chronic CRPS symptoms lasting more than 3 months [61]. A very recent retrospective cohort study of 39 patients treated with a 28-day taper regimen of $60 \mathrm{mg}$ prednisone provided further support for the use of a short course of prednisone to improve functionality in patients with CRPS [62].

Bisphosphonates are commonly used in the treatment of CRPS, largely due to evidence from several small RCTs that have shown significant positive effects $[57,63,64]$. Although the exact mechanism is not well understood, current research suggests bisphosphonates play a role in modulating inflammatory mediators, as well as 
the proliferation and migration of bone marrow cells $[8,9]$. The most recent RCT compared the use of three infusions of $60 \mathrm{mg}$ intravenous pamidronate to oral prednisolone in 21 poststroke patients with CRPS and found that pamidronate was just as effective for pain control [65]. A recent meta-analysis of four RCTs including a total of 181 patients showed a significant reduction of pain in patients with CRPS-1 taking bisphosphonates compared to placebo, demonstrating the efficacy and safety of bisphosphonates in the treatment of CRPS [66].

Gabapentin has also shown efficacy in reducing pain in patients with CRPS, although there are limited recent studies [57, 67, 68]. A small RCT compared the use of gabapentin to amitriptyline in children with CRPS-I or a neuropathic pain condition and found a significant reduction in pain score that did not differ between the medications [69]. With its favorable safety profile, the use of gabapentin for treating CRPS is largely dependent on provider preference and clinical experience [67].

The use of ketamine in the treatment of CRPS targets the sensitization of $\mathrm{N}$-methyl-Daspartic acid (NMDA) nociceptive pathways as a result of the upregulated inflammatory response $[5,70,71]$. By using an NMDA receptor antagonist, topical and intravenous ketamine have been shown in multiple placebo-controlled studies to be effective at providing pain relief and even inducing remission in treatment-resistant patients, although systematic reviews have concluded to be low-quality evidence overall [64, 72-75]. However, no recent large, RCTs have been conducted to further elucidate the efficacy, likely hindered by the frequent associated side effects [76].

Many antioxidants have been proposed for the treatment of CRPS based on the concept that local inflammation in CRPS generates oxygen free radicals [77]. However, vitamin $\mathrm{C}$ is the only antioxidant therapy supported by current evidence and is commonly used perioperatively for the prevention of CRPS following extremity surgery [78-80]. The most recent meta-analysis, which included three RCTs with a total of 875 patients, demonstrated a significant decrease in 1-year risk of CRPS after wrist fracture in patients taking daily $500 \mathrm{mg}$ vitamin C supplements for 50 days [80].

In recent years, as research has expanded our current understanding of the molecular and cellular mechanisms underlying the development of CRPS, a variety of new pharmacotherapies have been investigated in an attempt to create a more targeted approach. Naltrexone, a reversible competitive antagonist at $\mu$ - and $\kappa$ opioid receptors, has recently gained attention as a promising nonopioid modality for treating chronic, nonmalignant pain syndromes. In low doses, naltrexone acts primarily through an alternate pharmacodynamic pathway and inhibits microglial activation, ultimately decreasing the inflammatory response [81]. Low-dose naltrexone has commonly been used for off-label treatment of autoimmune diseases, such as multiple sclerosis and inflammatory bowel disease [81]. Limited data exist, which are primarily case studies, regarding its use in CRPS or other chronic pain syndromes, such as fibromyalgia [81-83]. As an inexpensive medication with a relatively benign adverse effect profile, further clinical research is warranted.

Another new area of research is the use of Botulinum toxin A (BTX-A), which has been used to alleviate pain in certain musculoskeletal disorders and off-label to treat neuropathic pain [84, 85]. Previous evidence was limited to case studies that used proximal injections of BTX-A for patients with CRPS related to myofascial pain syndrome (MFPS) [86, 87]. However, a recent study of 20 patients with refractory CRPS who received BTX-A injections demonstrated a significant reduction in pain scores [88]. Additionally, a very recent meta-analysis of 25 RCTs concluded that BTX-A is effective at reducing pain in both muscle-based (e.g., spasticity and dystonia) and non-muscle-based pain conditions, such as CRPS and diabetic neuropathy, further supporting the use of BTX-A as an alternative new treatment [85].

Recent advancements in the understanding of the autoimmune pathophysiology of CRPS have highlighted the potential benefits of plasma exchange therapy, which has historically treated other autoimmune conditions. A 2015 retrospective case series demonstrated that out of 33 patients who received plasma 
exchange therapy, 91\% reported a significant reduction in pain, with $45 \%$ reporting sustained pain relief with additional weekly treatment [89]. These preliminary findings support the need for larger RCTs to be performed in the future to further elucidate the efficacy of plasma exchange therapy.

The use of medical cannabis-based treatments for chronic pain disorders has received increasing research interest in recent years. However, several systematic reviews and metaanalyses have demonstrated conflicting results on efficacy and precise dosing remains a major challenge for clinicians to prescribe treatment regimens [90-94]. Additionally, legal considerations, optimal route of administration, and short-term adverse side effects, such as dizziness, nausea, and vomiting, have limited widespread use as a treatment modality [92, 95]. A very recent randomized, double-blinded, placebo-controlled trial demonstrated a significant and clinically meaningful reduction in pain after the use of a metered-dose cannabis inhaler compared to placebo in patients with chronic pain, including CRPS [95]. Given the precise low dose required and the practical advantages of inhaler administration, cannabis-based therapies are a promising new area of CRPS treatment that can be individualized to each patient [95]. Further studies will be needed to expand on the safety and efficacy of these regimens.

\section{Minimally Invasive/Interventional Therapy Treatments}

\section{Sympathetic Block}

A routinely used minimally invasive treatment for CRPS is a sympathetic block. Though routinely used, there is not a large amount of evidence on the short- and long-term analgesic effects of sympathetic blocks. The role of sympathetic blocks and epidural catheters and continuous sympathetic blocks has been described by Weissman et al., but Zernikow et al. concluded that there is a weak level of evidence for use of invasive treatments for CRPS in pediatric patients $[14,96]$. A study was performed in which after exclusions there were 318 patients, of those 318 patients 255 were diagnosed with CPRS (80\%); 155 of those CPRS patients received a pain reduction above $50 \%$. Many of these patients (71\%) experienced relief from pain for 1-4 weeks with a smaller percentage $(14 \%)$ who experienced relief for more than a month [97]. These data prove clinically significant and points to supporting the use of a sympathetic block for treatment whereas before there was a lack of evidence for this conclusion [98].

\section{Transcranial Magnetic Stimulation}

Another treatment that has seen success and may have a clinical application is transcranial magnetic stimulation (TMS). It is a safe and non-invasive technique that produces a brief magnetic pulse into the brain and can induce cortical excitability [99]. The research showed that this high-frequency stimulation had effect $30 \mathrm{~s}$ after treatment and continued to reduce pain beyond 1 week [99]. More research on the long-term pain relief is needed to be able to make a conclusive statement on the efficacy of TMS.

\section{Surgical Management}

Several options have been used as surgical treatment for CRPS in effort to avoid excessive use of opioids and improve quality of life. One such therapy is spinal cord stimulation, which has been used for over 50 years as the most common treatment for chronic pain globally [100]. This approach traditionally involves tonic electrical stimulation of the dorsal columns avoiding ablation and in theory utilizing the 'gate-control' theory of Melzack and Wall $[100,101]$. Other explanations for effectiveness include modulating neuronal hyperexcitability and neurotransmitter concentration [100]. Additionally, SCS has been shown to affect protein levels in the cerebrospinal fluid (CSF) as neuroprotection, which indicates benefits beyond the effect on neurotransmitter concentration [100, 102]. These levels of CSF proteins act by modifying nociceptive signals, immune regulation, and neuroplasticity as well $[100,102]$. Furthermore, effects of high-frequency SCS at $10 \mathrm{kHz}$ (HF10-SCS) has been shown to significantly improve symptoms with 
most participants reporting over 50\% improvement during trials [103]. Encouragingly, the majority in the study with CRPS and previous attempt with traditional SCS without relief or who were no longer receiving relief did obtain improvement [103]. HF10-SCS has been described as a rescue therapy for those in this cohort [103]. A postulated mechanism for the HF10SCS benefit as an adjunct to traditional SCS is direct inhibition of wide dynamic range neurons and signal modulation [103]. Common criticism of traditional SCS involves reliance on paresthesia, but HF10-SCS aims to solve this problem [100, 103]. Overall, SCS is considered effective and advisable, especially for CRPS patients who have undergone 12-16 weeks of conservative treatment without significant effect [100].

Another surgical treatment that exists for patients with CRPS is implantable peripheral nerve stimulation (PNS). This exists as a more focal approach to specific nerves that have presented problematically with regards to response. One study utilized a surgical procedure that addressed specific neuropathy in upper and lower extremity nerves with the sciatic nerve being the most commonly affected in this cohort [104]. Significant improvements were seen in visual analog scale (VAS) pain rating, reduction of patients on concurrent opioid therapy, and improved functional outcomes [104]. Interestingly, many included in this study also featured implantable SCS, which highlights PNS as adjunct treatment [104]. Another study points to more proximal PNS option by targeting the brachial plexus for specific upper extremity problems, which remain the majority $[104,105]$. A similar study showed significant improvement in VAS, the Neuropathic Pain Scale (NPS), as well as functional improvement as measured by Short-Form Health Survey 12-item (SF-12) [105].

Intermediate to the previously mentioned surgical interventions lies dorsal root ganglion (DRG) stimulation. A large, randomized trial comparing DRG stimulation to SCS showed higher rates of treatment success and longer persistence of effects using DRG stimulation [106]. It was shown that safety was comparable and DRG stimulation had improved quality of life, psychological outlook, and significantly less postural-related changes in paresthesia when compared with SCS [106]. It is suggested that direct effect of stimulation involving specific dermatomal patterns lends to the success of DRG stimulation in specific targeting [106].

Amputation has long been employed and is among the most controversial surgical treatments for CRPS. A systematic review found that although prominent risks of phantom limb pain (PLP) and recurrence are of consideration, there exist many studies showing possible improvement of symptoms with amputation [107]. Stoehr et al. in their 2020 article in Microsurgery treated four patients ranging in age from 38 to 71 years with targeted muscle reinnervation [108]. Two of the subjects developed residual limb pain (RLP) and PLP, one only had RLP, and one only had PLP [108]. Due to the conflicting results and historical avoidance, some of the most important aspects of care that improve surgical outcomes include pre-, peri-, and postoperative care irrespective of procedure performed [107, 109]. A team approach using accurate diagnosis, selection of procedure, and appropriate followed care are critical for successful outcomes [109].

\section{Future Therapy}

With the expanding knowledge base of medicine, the future holds many novel and promising treatments. One such treatment includes use of mycophenolate, which has historical use as an immunosuppressant and anecdotal evidence that it improves neuropathic pain [110]. A study showed intriguing results, prompting additional studies on immunosuppressantbased treatment for analgesic effects. However, considerations exist due to the unknown mechanism, inconsistent results, and possible side-effect profiles [110]. Similarly, a different study examined use of another substance, polydeoxyribonucleotide (PDRN), which has anti-inflammatory and regenerative effects [111]. Results did show some allodynia reduction effects along with activation of astrocytes in animal models, which begs future 
investigation [111]. Finally, as perhaps the most novel of applications, immersive virtual reality has been applied to reduce neuropathic pain in CRPS [112]. This pilot study of eight participants used a kitchen simulation software and discovered that patients with upper-extremity CRPS who completed at least ten sessions showed some improvement [112]. Chau et al. reported that participants described pain relief and functional improvement possibly using a similar mechanism of effect as mirror therapy stimulating mirror neurons and neuroplasticity [112]. These innovative future therapies need more examination to prove effectiveness and longevity, but all are encouraging at this juncture.

\section{CONCLUSIONS}

CRPS is a complex and multifactorial condition. While our current understanding of CRPS has come a long way since those early definitions, it is still not complete. Larger and higher-quality clinical studies are needed to further elucidate the underlying mechanisms of this condition, which will enable the development of more precisely targeted therapies. Although advances in novel treatments have expanded the range of therapy options, no successful therapeutic intervention exists. Therefore, continued research efforts are needed to investigate combinations of medical and surgical therapies for the future of CRPS treatment.

\section{ACKNOWLEDGEMENTS}

Funding. No funding or sponsorship was received for this study or for publication of this article.

Editorial Assistance. The authors are grateful to the Paolo Procacci Foundation for editing support.

Authorship. All named authors meet the International Committee of Medical Journal Editors (ICMJE) criteria for authorship for this article, take responsibility for the integrity of the work as a whole, and have given their approval for this version to be published.

Authors' Contributions. All authors have contributed to review and ameliorate the quality of the paper and have reviewed and approved the final draft of the manuscript.

Disclosures. Samantha-Su Taylor, Nazir Noor, Ivan Urits, Monica Sri Sadhu, Clay Gibb, Tyler Carlson, Dariusz Myrcik, and Omar Viswanath have nothing to disclose. Antonella Paladini is a member of the journal's Editorial Board. Giustino Varrassi is the Editor-in-Chief of the journal.

Compliance with Ethics Guidelines. This article is based on previously conducted studies and does not contain any studies with human participants or animal performed by the authors without a previous Ethics Committee approval.

Open Access. This article is licensed under a Creative Commons Attribution-NonCommercial 4.0 International License, which permits any non-commercial use, sharing, adaptation, distribution and reproduction in any medium or format, as long as you give appropriate credit to the original author(s) and the source, provide a link to the Creative Commons licence, and indicate if changes were made. The images or other third party material in this article are included in the article's Creative Commons licence, unless indicated otherwise in a credit line to the material. If material is not included in the article's Creative Commons licence and your intended use is not permitted by statutory regulation or exceeds the permitted use, you will need to obtain permission directly from the copyright holder. To view a copy of this licence, visit http://creativecommons.org/licenses/by$\mathrm{nc} / 4.0 /$.

\section{REFERENCES}

1. Ott S, Maihöfner C. Signs and symptoms in 1,043 patients with complex regional pain syndrome. 
J Pain. 2018;19(6):599-611. https://doi.org/10. 1016/j.jpain.2018.01.004.

2. Mertz K, Trunzter J, Wu E, Barnes J, Eppler SL, Kamal RN. National trends in the diagnosis of CRPS after open and endoscopic carpal tunnel release. J Wrist Surg. 2019;08(03):209-14. https://doi.org/ 10.1055/s-0039-1678674.

3. Jo Y-H, Kim K, Lee B-G, Kim J-H, Lee C-H, Lee K-H. Incidence of and risk factors for complex regional pain syndrome type 1 after surgery for distal radius fractures: a population-based study. Sci Rep. 2019. https://doi.org/10.1038/S41598-019-41152-X.

4. Marinus J, Moseley GL, Birklein F, et al. Clinical features and pathophysiology of complex regional pain syndrome. Lancet Neurol. 2011;10(7):637-48. https://doi.org/10.1016/S1474-4422(11)70106-5.

5. Goh EL, Chidambaram S, Ma D. Complex regional pain syndrome: a recent update. Burn Trauma. 2017. https://doi.org/10.1186/s41038-016-0066-4.

6. Eldufani J, Elahmer N, Blaise G. A medical mystery of complex regional pain syndrome. Heliyon. 2020;6(2):e03329. heliyon.2020.e03329.

7. Shim H, Rose J, Halle S, Shekane P. Complex regional pain syndrome: a narrative review for the practising clinician. Br J Anaesth. 2019;123(2): e424-33. https://doi.org/10.1016/j.bja.2019.03.030.

8. Urits I, Shen AH, Jones MR, Viswanath O, Kaye AD. Complex regional pain syndrome, current concepts and treatment options. Curr Pain Headache Rep. 2018. https://doi.org/10.1007/s11916-018-0667-7.

9. Misidou C, Papagoras C. Complex regional pain syndrome: an update. Mediterr J Rheumatol. 2019;30(1):16-25. https://doi.org/10.31138/mjr.30. 1.16 .

10. Elsharydah A, Loo NH, Minhajuddin A, Kandil ES. Complex regional pain syndrome type 1 predictors-epidemiological perspective from a national database analysis. J Clin Anesth. 2017. https://doi. org/10.1016/j.jclinane.2017.03.027.

11. Murphy KR, Han JL, Yang S, Hussaini SM, Elsamadicy AA, Parente B, Xie J, Pagadala LS. Prevalence of specific types of pain diagnoses in a sample of United States adults. Pain Physician. 2017;20(2): E257-68.

12. Elsamadicy AA, Yang $S$, Sergesketter AR, et al. Prevalence and cost analysis of complex regional pain syndrome (CRPS): a role for neuromodulation. Neuromodulation. 2018;21(5):423-30. https://doi. org/10.1111/ner.12691.
13. Karri J, Palmer JS, Charnay A, et al. Utility of electrical neuromodulation for treating chronic pain syndromes in the pediatric setting: a systematic review. Neuromodulation. 2021. https://doi.org/10. 1111/ner.13365.

14. Weissmann R, Uziel Y. Pediatric complex regional pain syndrome: a review. Pediatr Rheumatol. 2016. https://doi.org/10.1186/s12969-016-0090-8.

15. Wiper A, Amoroso G, Rao S, Nolan J. Complex regional pain syndrome: a rare but potentially disabling complication of transradial cardiac catheterization. Catheter Cardiovasc Interv. 2020;95(5):E140-3. https://doi.org/10.1002/ccd. 28357.

16. Crijns TJ, Van Der Gronde BATD, Ring D, Leung N. Complex regional pain syndrome after distal radius fracture is uncommon and is often associated with fibromyalgia. Clin Orthop Relat Res. 2018;476(4): 744-50. https://doi.org/10.1007/s11999. 0000000000000070 .

17. Rochlin DH, Sheckter CC, Satteson ES, Swan CC, Fox PM, Curtin C. Separating fact from fiction: a nationwide longitudinal examination of complex regional pain syndrome following treatment of Dupuytren contracture. Hand. 2020. https://doi. org/10.1177/1558944720963915.

18. van Velzen GAJ, Huygen FJPM, van Kleef M, van Eijs FV, Marinus J, van Hilten JJ. Sex matters in complex regional pain syndrome. Eur J Pain (United Kingdom). 2019;23(6):1108-16. https://doi.org/10.1002/ ejp.1375.

19. Lipman MD, Hess DE, Werner BC, Deal DN. Fibromyalgia as a predictor of complex regional pain syndrome after distal radius fracture. Hand. 2019;14(4):516-22. https://doi.org/10.1177/ 1558944717735949.

20. Birklein F, Ajit SK, Goebel A, Perez RSGM, Sommer C. Complex regional pain syndrome-phenotypic characteristics and potential biomarkers. Nat Rev Neurol. 2018;14(5):272-84. https://doi.org/10. 1038/nrneurol.2018.20.

21. Ward D, Thorsen NM, Frisch M, Valentiner-Branth P, Mølbak K, Hviid A. A cluster analysis of serious adverse event reports after human papillomavirus (HPV) vaccination in Danish girls and young women, September 2009 to August 2017. Euro Surveill. 2019;24(19):1. https://doi.org/10.2807/15607917.ES.2019.24.19.1800380.

22. Hviid A, Thorsen NM, Valentiner-Branth P, Frisch M, Mølbak K. Association between quadrivalent human papillomavirus vaccination and selected syndromes with autonomic dysfunction in Danish females: population based, self-controlled, case 
series analysis. BMJ. 2020. https://doi.org/10.1136/ bmj.m2930.

23. Barboi A, Gibbons CH, Axelrod F, et al. Human papillomavirus (HPV) vaccine and autonomic disorders: a position statement from the American Autonomic Society. Auton Neurosci Basic Clin. 2020;223(1):13-8. https://doi.org/10.1016/j.autneu. 2019.05.002.

24. Baronio M, Sadia H, Paolacci S, et al. Molecular aspects of regional pain syndrome. Pain Res Manag. 2020. https://doi.org/10.1155/2020/7697214.

25. Li WW, Guo TZ, Shi X, et al. Neuropeptide regulation of adaptive immunity in the tibia fracture model of complex regional pain syndrome. J Neuroinflamm. 2018;15(1):105. https://doi.org/10. 1186/s12974-018-1145-1.

26. Dirckx M, Stronks DL, Van Bodegraven-Hof EAM, Wesseldijk F, Groeneweg JG, Huygen FJPM. Inflammation in cold complex regional pain syndrome. Acta Anaesthesiol Scand. 2015;59(6):733-9. https://doi.org/10.1111/aas.12465.

27. Sommer C, Leinders M, Üçeyler N. Inflammation in the pathophysiology of neuropathic pain. Pain. 2018;159(3):595-602. https://doi.org/10.1097/j. pain.0000000000001122.

28. Wehmeyer C, Pap T, Buckley CD, Naylor AJ. The role of stromal cells in inflammatory bone loss. Clin Exp Immunol. 2017;189(1):1-11. https://doi.org/ 10.1111/cei.12979.

29. Russo MA, Georgius P, Pires AS, et al. Novel immune biomarkers in complex regional pain syndrome. J Neuroimmunol. 2020;347:577330. https://doi. org/10.1016/j.jneuroim.2020.577330.

30. Bharwani KD, Dik WA, Dirckx M, Huygen FJPM. Highlighting the role of biomarkers of inflammation in the diagnosis and management of complex regional pain syndrome. Mol Diagn Ther. 2019;23(5):615-26. https://doi.org/10.1007/ s40291-019-00417-x.

31. Littlejohn G. Neurogenic neuroinflammation in fibromyalgia and complex regional pain syndrome. Nat Rev Rheumatol. 2015;11:639-48. https://doi. org/10.1038/nrrheum.2015.100.

32. Birklein F, Drummond PD, Li W, et al. Activation of cutaneous immune responses in complex regional pain syndrome. J Pain. 2014;15(5):485-95. https:// doi.org/10.1016/j.jpain.2014.01.490.

33. Yvon A, Faroni A, Reid AJ, Lees VC. Selective fiber degeneration in the peripheral nerve of a patient with severe complex regional pain syndrome. Front
Neurosci. 2018;12(APR):207. https://doi.org/10. 3389/fnins.2018.00207.

34. Knudsen LF, Terkelsen AJ, Drummond PD, Birklein F. Complex regional pain syndrome: a focus on the autonomic nervous system. Clin Auton Res. 2019;29(4):457-67. https://doi.org/10.1007/ s10286-019-00612-0.

35. Kortekaas MC, Niehof SP, Stolker RJ, Huygen FJPM. Pathophysiological mechanisms involved in vasomotor disturbances in complex regional pain syndrome and implications for therapy: a review. Pain Pract. 2016;16(7):905-14. https://doi.org/10.1111/ papr.12403.

36. Drummond PD, Morellini N, Finch PM, Birklein F, Knudsen LF. Complex regional pain syndrome. Pain. 2018;159(11):2296-305. https://doi.org/10. 1097/j.pain.0000000000001335.

37. Chen S-S, Zhang J-M. Progress in sympathetically mediated pathological pain. J Anesth Perioper Med. 2015;2(4):216-25. https://doi.org/10.24015/japm. 2015.0029 .

38. Xie W, Strong JA, Zhang JM. Local knockdown of the NaV1.6 sodium channel reduces pain behaviors, sensory neuron excitability, and sympathetic sprouting in rat models of neuropathic pain. Neuroscience. 2015;291:317-30. https://doi.org/10. 1016/j.neuroscience.2015.02.010.

39. Tajerian M, Clark JD. New concepts in complex regional pain syndrome. Hand Clin. 2016;32(1): 41-9. https://doi.org/10.1016/j.hcl.2015.08.003.

40. Cuhadar U, Gentry C, Vastani N, et al. Autoantibodies produce pain in complex regional pain syndrome by sensitizing nociceptors. Pain. 2019;160(12):2855-65. https://doi.org/10.1097/j. pain. 0000000000001662 .

41. David Clark J, Tawfik VL, Tajerian M, Kingery WS. Autoinflammatory and autoimmune contributions to complex regional pain syndrome. Mol Pain. 2018. https://doi.org/10.1177/1744806918799127.

42. Dubuis E, Thompson V, Leite MI, et al. Longstanding complex regional pain syndrome is associated with activating autoantibodies against alpha-1a adrenoceptors. Pain. 2014;155(11):2408-17. https:// doi.org/10.1016/j.pain.2014.09.022.

43. Kohr D, Singh P, Tschernatsch M, et al. Autoimmunity against the $\beta 2$ adrenergic receptor and muscarinic-2 receptor in complex regional pain syndrome. Pain. 2011;152(12):2690-700. https:// doi.org/10.1016/j.pain.2011.06.012.

44. Tan W, Song Y, Mo C, Jiang S, Wang Z. Analysis of gene expression profile microarray data in complex 
regional pain syndrome. Mol Med Rep. 2017;16(3): 3371-8. https://doi.org/10.3892/mmr.2017.6950.

45. Bruehl S, Gamazon ER, Van De Ven T, et al. DNA methylation profiles are associated with complex regional pain syndrome after traumatic injury HHS Public Access. Pain. 2019;160(10):2328-37. https:// doi.org/10.1097/j.pain.0000000000001624.

46. Van Rooijen DE, Roelen DL, Verduijn W, et al. Genetic HLA associations in complex regional pain syndrome with and without dystonia. J Pain. 2012;13(8):784-9. https://doi.org/10.1016/j.jpain. 2012.05.003.

47. D’Agnelli S, Gerra MC, Bignami E, Arendt-Nielsen L. Exosomes as a new pain biomarker opportunity. Mol Pain. 2020;16:1-9. https://doi.org/10.1177/ 1744806920957800 .

48. Park HY, Jang YE, Oh S, Lee PB. Psychological characteristics in patients with chronic complex regional pain syndrome: comparisons with patients with major depressive disorder and other types of chronic pain. J Pain Res. 2020;13:389-98. https:// doi.org/10.2147/JPR.S230394.

49. Speck V, Schlereth T, Birklein F, Maihöfner C. Increased prevalence of posttraumatic stress disorder in CRPS. Eur J Pain. 2017;21(3):466-73. https:// doi.org/10.1002/ejp.940.

50. Halicka M, Vittersø AD, Proulx MJ, Bultitude JH. Neuropsychological changes in complex regional pain syndrome (CRPS). Behav Neurol. 2020;2020: 1-30. https://doi.org/10.1155/2020/4561831.

51. Harden RN, Bruehl S, Perez RSGM, et al. Validation of proposed diagnostic criteria (the "Budapest Criteria") for complex regional pain syndrome. Pain. 2010;150(2):268-74. https://doi.org/10.1016/j.pain. 2010.04.030.

52. Baygutalp F, Kul A. Effect of early orthopedic rehabilitation on development of complex regional pain syndrome type 1. Eurasian J Med. 2020;52(2):110-4. https://doi.org/10.5152/eurasianjmed.2020.19231.

53. Lewis JS, Kellett S, McCullough R, et al. Body perception disturbance and pain reduction in longstanding complex regional pain syndrome following a multidisciplinary rehabilitation program. Pain Med (United States). 2019;20(11): 2213-9. https://doi.org/10.1093/pm/pnz176.

54. Smart KM, Wand BM, O'Connell NE. Physiotherapy for pain and disability in adults with complex regional pain syndrome (CRPS) types I and II. Cochrane Database Syst Rev. 2016. https://doi.org/ 10.1002/14651858.CD010853.pub2.
55. Méndez-Rebolledo G, Gatica-Rojas V, Torres-Cueco R, Albornoz-Verdugo M, Guzmán-Muñoz E. Update on the effects of graded motor imagery and mirror therapy on complex regional pain syndrome type 1: a systematic review. J Back Musculoskelet Rehabil. 2017;30(3):441-9. https://doi.org/10.3233/BMR150500 .

56. McGee C, Skye J, Van Heest A. Graded motor imagery for women at risk for developing type I CRPS following closed treatment of distal radius fractures: a randomized comparative effectiveness trial protocol. BMC Musculoskelet Disord. 2018. https://doi. org/10.1186/s12891-018-2115-6.

57. Wertli MM, Kessels AGH, Perez RSGM, Bachmann LM, Brunner F. Rational pain management in complex regional pain syndrome 1 (CRPS 1)-a network meta-analysis. Pain Med (United States). 2014;15(9):1575-89. https://doi.org/10.1111/pme. 12466.

58. Krumova E, Breuer AJ, Mainka T, Hansel N, Maier C, Krumova EK. Short-term treatment with parecoxib for complex regional pain syndrome: a randomized, placebo-controlled double-blind trial. Pain Physician. 2014;17:127-37.

59. Eraghi AS, Khazanchin A, Hosseinzadeh N, Pahlevansabagh A. A randomized controlled trial on Aspirin and complex regional pain syndrome after radius fractures. Eur J Transl Myol. 2020;30(1):1-8. https://doi.org/10.4081/ejtm.2019.8643.

60. Kalita J, Vajpayee A, Misra UK. Comparison of prednisolone with piroxicam in complex regional pain syndrome following stroke: a randomized controlled trial. QJM Mon J Assoc Physicians. 2006;99(2):89-95. https://doi.org/10.1093/qjmed/ hcl004.

61. Barbalinardo S, Loer SA, Goebel A, Perez RSGM. The treatment of longstanding complex regional pain syndrome with oral steroids. Pain Med. 2016;17(2): 337-43. https://doi.org/10.1093/pm/pnv002.

62. Jamroz A, Berger M, Winston P. Prednisone for acute complex regional pain syndrome: a retrospective cohort study. Pain Res Manag. 2020. https://doi.org/10.1155/2020/8182569.

63. Żyluk A, Puchalski P. Effectiveness of complex regional pain syndrome treatment: a systematic review. Neurol Neurochir Pol. 2018;52(3):326-33. https://doi.org/10.1016/j.pjnns.2018.03.001.

64. Xu J, Yang J, Lin P, Rosenquist E, Cheng J. Intravenous therapies for complex regional pain syndrome. Anesth Analg. 2016;122(3):843-56. https:// doi.org/10.1213/ANE.0000000000000999. 
65. Young HE, Hyeyun K, Hee IS. Pamidronate effect compared with a steroid on complex regional pain syndrome type I: pilot randomised trial. Neth J Med. 2016;74(1):30-5.

66. Chevreau M, Romand X, Gaudin P, Juvin R, Baillet A. Bisphosphonates for treatment of Complex Regional Pain Syndrome type 1: a systematic literature review and meta-analysis of randomized controlled trials versus placebo. Jt Bone Spine. 2017;84(4):393-9. https://doi.org/10.1016/j.jbspin. 2017.03.009.

67. Wiffen PJ, Derry S, Bell RF, et al. Gabapentin for chronic neuropathic pain in adults. Cochrane Database Syst Rev. 2017. https://doi.org/10.1002/ 14651858.CD007938.pub4.

68. O'Connell NE, Wand BM, Mcauley J, Marston L, Moseley GL. Interventions for treating pain and disability in adults with complex regional pain syndrome- an overview of systematic reviews. Cochrane Database Syst Rev. 2013. https://doi.org/ 10.1002/14651858.CD009416.pub2.

69. Brown S, Johnston B, Amaria K, et al. A randomized controlled trial of amitriptyline versus gabapentin for complex regional pain syndrome type I and neuropathic pain in children. Scand J Pain. 2016;13: 156-63. https://doi.org/10.1016/j.sjpain.2016.05. 039.

70. Yang Y, Maher DP, Cohen SP. Emerging concepts on the use of ketamine for chronic pain. Expert Rev Clin Pharmacol. 2020;13(2):135-46. https://doi. org/10.1080/17512433.2020.1717947.

71. Schwartzman RJ, Alexander GM, Grothusen JR. The use of ketamine in complex regional pain syndrome: possible mechanisms. Expert Rev Neurother. 2011;11(5):719-34. https://doi.org/10.1586/ ern.11.31.

72. Zhao J, Wang Y, Wang D. The Effect of ketamine infusion in the treatment of complex regional pain syndrome: a systemic review and meta-analysis. Curr Pain Headache Rep. 2018;22(2):1-8. https:// doi.org/10.1007/s11916-018-0664-X.

73. Finch PM, Knudsen L, Drummond PD. Reduction of allodynia in patients with complex regional pain syndrome: a double-blind placebo-controlled trial of topical ketamine. Pain. 2009;146(1-2):18-25. https://doi.org/10.1016/j.pain.2009.05.017.

74. Schwartzman RJ, Alexander GM, Grothusen JR, Paylor T, Reichenberger E, Perreault M. Outpatient intravenous ketamine for the treatment of complex regional pain syndrome: a double-blind placebocontrolled study. Pain. 2009;147(1-3):107-15. https://doi.org/10.1016/j.pain.2009.08.015.
75. Kiefer RT, Rohr P, Ploppa A, et al. Efficacy of ketamine in anesthetic dosage for the treatment of refractory complex regional pain syndrome: an open-label phase II study. Pain Med. 2008;9(8): 1173-201. https://doi.org/10.1111/j.1526-4637. 2007.00402.x.

76. Azari P, Lindsay DR, Briones D, Clarke C, Buchheit T, Pyati S. Efficacy and safety of ketamine in patients with complex regional pain syndrome: a systematic review. CNS Drugs. 2012;26(3):215-28. https://doi.org/10.2165/11595200-00000000000000 .

77. Perez RSGM, Zuurmond WWA, Bezemer PD, et al. The treatment of complex regional pain syndrome type I with free radical scavengers: a randomized controlled study. Pain. 2003;102(3):297-307. https://doi.org/10.1016/S0304-3959(02)00414-1.

78. Chen S, Roffey DM, Dion CA, Arab A, Wai EK. Effect of perioperative Vitamin $C$ supplementation on postoperative pain and the incidence of chronic regional pain syndrome: a systematic review and meta-analysis. Clin J Pain. 2016;32(2):179-85. https://doi.org/10.1097/AJP.0000000000000218.

79. Meena S, Sharma P, Gangary SK, Chowdhury B. Role of vitamin $\mathrm{C}$ in prevention of complex regional pain syndrome after distal radius fractures: a meta-analysis. Eur J Orthop Surg Traumatol. 2015;25(4):637-41. https://doi.org/10.1007/ s00590-014-1573-2.

80. Aïm F, Klouche S, Frison A, Bauer T, Hardy P. Efficacy of vitamin $\mathrm{C}$ in preventing complex regional pain syndrome after wrist fracture: a systematic review and meta-analysis. Orthop Traumatol Surg Res. 2017;103(3):465-70. https://doi.org/10.1016/j. otsr.2016.12.021.

81. Trofimovitch D, Baumrucker SJ. Pharmacology update: low-dose naltrexone as a possible nonopioid modality for some chronic, nonmalignant pain syndromes. Am J Hosp Palliat Med. 2019;36(10): 907-12. 1049909119838974.

https://doi.org/10.1177/

82. Chopra P, Cooper MS. Treatment of complex regional pain syndrome (CRPS) using low dose naltrexone (LDN). J Neuroimmune Pharmacol. 2013;8(3):470-6. https://doi.org/10.1007/s11481013-9451-y.

83. Younger J, Mackey S. Fibromyalgia symptoms are reduced by low-dose naltrexone: a pilot study. Pain Med. 2009;10(4):663-72. https://doi.org/10.1111/j. 1526-4637.2009.00613.x.

84. Argoff CE. A focused review on the use of botulinum toxins for neuropathic pain. Clin J Pain. 2002. 
https://doi.org/10.1097/00002508-20021100100010.

85. Siongco PRL, Rosales RL, Moore AP, et al. Botulinum neurotoxin injections for muscle-based (dystonia and spasticity) and non-muscle-based (neuropathic pain) pain disorders: a meta-analytic study. J Neural Transm. 2020;127(6):935-51. https://doi.org/10.1007/s00702-020-02163-5.

86. Birthi P, Sloan P, Salles S. Subcutaneous botulinum toxin A for the treatment of refractory complex regional pain syndrome. PM\&R. 2012;4(6):446-9. https://doi.org/10.1016/j.pmrj.2011.12.010.

87. Safarpour D, Jabbari B. Botulinum toxin A (BOTOX) for treatment of proximal myofascial pain in complex regional pain syndrome: two cases. Pain Med. 2010;11(9):1415-8. https://doi.org/10.1111/j.15264637.2010.00929.x.

88. Lessard L, Bartow MJ, Lee J, Diaz-Abel J, Tessler OES. Botulinum toxin A. Plast Reconstr Surg Glob Open. 2018;6(10):e1847. https://doi.org/10.1097/GOX. 0000000000001847.

89. Alexander GM, Aradillas E, Schwartzman RJ, Grothusen JR, Goebel A. Retrospective study plasma exchange therapy in patients with complex regional pain syndrome. Pain Physician. 2015;18:383-94.

90. Hill KP. Medical marijuana for treatment of chronic pain and other medical and psychiatric problems: a clinical review. JAMA J Am Med Assoc. 2015;313(24):2474-83. https://doi.org/10.1001/ jama.2015.6199.

91. Andreae MH, Carter GM, Shaparin N, et al. Inhaled cannabis for chronic neuropathic pain: a metaanalysis of individual patient data. J Pain. 2015;16(12):1221-32. https://doi.org/10.1016/j. jpain.2015.07.009.

92. Whiting PF, Wolff RF, Deshpande $\mathrm{S}$, et al. Cannabinoids for medical use: a systematic review and meta-analysis. JAMA J Am Med Assoc. 2015;313(24):2456-73. https://doi.org/10.1001/ jama.2015.6358.

93. Meng H, Johnston B, Englesakis M, Moulin DE, Bhatia A. Selective cannabinoids for chronic neuropathic pain: a systematic review and meta-analysis. Anesth Analg. 2017;125(5):1638-52. https://doi. org/10.1213/ANE.0000000000002110.

94. Stockings E, Campbell G, Hall WD, et al. Cannabis and cannabinoids for the treatment of people with chronic noncancer pain conditions: a systematic review and meta-analysis of controlled and observational studies. Pain. 2018;159(10):1932-54. https://doi.org/10.1097/j.pain.0000000000001293.
95. Almog S, Aharon-Peretz J, Vulfsons S, et al. The pharmacokinetics, efficacy, and safety of a novel selective-dose cannabis inhaler in patients with chronic pain: a randomized, double-blinded, placebo-controlled trial. Eur J Pain (United Kingdom). 2020;24(8):1505-16. https://doi.org/10.1002/ejp. 1605.

96. Zernikow B, Wager J, Brehmer H, Hirschfeld G, Maier C. Invasive treatments for complex regional pain syndrome in children and adolescents: a scoping review. Anesthesiology. 2015;122(3): 699-707. https://doi.org/10.1097/ALN. 0000000000000573.

97. Cheng J, Salmasi V, You J, et al. Outcomes of sympathetic blocks in the management of complex regional pain syndrome: a retrospective cohort study. Anesthesiology. 2019;131(4):883-93. https:// doi.org/10.1097/ALN.0000000000002899.

98. O'Connell NE, Wand BM, Gibson W, Carr DB, Birklein F, Stanton TR. Local anaesthetic sympathetic blockade for complex regional pain syndrome. Cochrane Database Syst Rev. 2016;2016(7): 1-57. https://doi.org/10.1002/14651858. CD004598.pub4.

99. Yang S, Chang MC. Effect of repetitive transcranial magnetic stimulation on pain management: a systematic narrative review. Front Neurol. 2020;11: 114. https://doi.org/10.3389/fneur.2020.00114.

100. Isagulyan E, Slavin K, Konovalov N, et al. Spinal cord stimulation in chronic pain: technical advances. Korean J Pain. 2020;33(2):99-107. https://doi. org/10.3344/kjp.2020.33.2.99.

101. Melzack R, Wall PD. Pain mechanisms: a new theory. Science (80-). 1965;150(3699):971-9. https:// doi.org/10.1126/science.150.3699.971.

102. Lind AL, Emami Khoonsari P, Sjödin M, et al. Spinal cord stimulation alters protein levels in the cerebrospinal fluid of neuropathic pain patients: a proteomic mass spectrometric analysis. Neuromodulation. 2016;19(6):549-62. https://doi. org/10.1111/ner.12473.

103. Gill JS, Asgerally A, Simopoulos TT. High-frequency spinal cord stimulation at $10 \mathrm{kHz}$ for the treatment of complex regional pain syndrome: a case series of patients with or without previous spinal cord stimulator implantation. Pain Pract. 2019;19(3): 289-94. https://doi.org/10.1111/papr.12739.

104. Chmiela MA, Hendrickson M, Hale J, et al. Direct peripheral nerve stimulation for the treatment of complex regional pain syndrome: a 30-year review. Neuromodulation. 2020. https://doi.org/10.1111/ ner.13295. 
105. Frederico TN, da Silva FT. Peripheral nerve stimulation of the brachial plexus for chronic refractory CRPS pain of the upper limb: description of a new technique and case series. Pain Med. 2020;21(1): S18-26. https://doi.org/10.1093/pm/pnaa201.

106. Deer TR, Levy RM, Kramer J, et al. Dorsal root ganglion stimulation yielded higher treatment success rate for complex regional pain syndrome and causalgia at 3 and 12 months: a randomized comparative trial. Pain. 2017;158(4):669-81. https://doi.org/10.1097/j.pain.0000000000000814.

107. Ayyaswamy B, Saeed B, Anand A, Chan L, Shetty V. Quality of life after amputation in patients with advanced complex regional pain syndrome: a systematic review. EFORT Open Rev. 2019;4(9): 533-40. https://doi.org/10.1302/2058-5241.4. 190008.

108. Stoehr JR, Sood R, Jordan SW, Dumanian GA. Targeted muscle reinnervation at the time of amputation in the management of complex regional pain syndrome of the lower extremity. Microsurgery.
2020;40(8):852-8. https://doi.org/10.1002/micr. 30653.

109. Poppler LH, Mackinnon SE. The role of the peripheral nerve surgeon in the treatment of pain. Neurotherapeutics. 2019;16(1):9-25. https://doi.org/10. 1007/s13311-018-00695-z.

110. Goebel A, Jacob A, Frank B, et al. Mycophenolate for persistent complex regional pain syndrome, a parallel, open, randomised, proof of concept trial. Scand J Pain. 2018;18(1):29-37. https://doi.org/10. 1515/sjpain-2017-0154.

111. Lee SH, Yoo SH, Lee HJ, et al. Anti-allodynic effects of polydeoxyribonucleotide in an animal model of neuropathic pain and complex regional pain syndrome. J Korean Med Sci. 2020. https://doi.org/10. 3346/JKMS.2020.35.E225.

112. Chau B, Phelan I, Ta $P$, et al. Immersive virtual reality for pain relief in upper limb complex regional pain syndrome: a pilot study. Innov Clin Neurosci. 2020;17(4-6):47-52. 